\title{
An Improved Characterization of Small Scale Fading Based on 2D Measurements and Modeling of a Moving Receiver in an Indoor Environment
}

\author{
Redhwan Mawari', Alexander H. Henderson², Muhammad B. Akbar ${ }^{3}$, \\ Gregory D. Durgin ${ }^{3}$, Mohamed Zohdy ${ }^{1}$ \\ ${ }^{1}$ Department of Electrical and Computer Engineering, Oakland University, Rochester, MI, USA \\ ${ }^{2}$ Rice University, Houston, TX, USA \\ ${ }^{3}$ Department of Electrical and Computer Engineering, Georgia Institute of Technology, Atlanta, GA, USA \\ Email: rmawari@oakland.edu, zohdyma@oakland.edu, ah1@alumni.rice.edu, \\ durgin@gatech.edu, makbar7@gatech.edu
}

Received 14 June 2016; accepted 8 August 2016; published 11 August 2016

Copyright (C) 2016 by authors and Scientific Research Publishing Inc.

This work is licensed under the Creative Commons Attribution International License (CC BY).

http://creativecommons.org/licenses/by/4.0/

(c) (i) Open Access

\section{Abstract}

Accurately characterizing the wireless small-scale fading channel has been a challenging task in the wireless communication era due to the surrounding environment. Therefore, this paper introduces a new technique to experimentally characterize the small-scale fading taking under consideration real environmental conditions. By conducting a two dimensional measurement while the mobile receiver is moving; a more accurate channel will be achieved. Two-dimensional measurement refers to collecting data from the receiver along the $x$ and $y$ direction. The two-dimensional measurement data contain far more information than a one-dimensional data collected. In order to represent the small-scale channel along with the real environmental conditions, new approaches are necessary to configure the two-dimensional system and to analyze the 2D data. The new approach this paper introduces for the characterization is that the measurements are conducted on a receiver while it is moving in a two dimensional manner, under different scenarios, Line-of-sight, Non-line-of-sight, and Two-wave-Diffuse Power. The experiment was conducted in a 7 meters long by 4 meters wide room, wherein the distance between the transmitter antenna and receiver is about 3 meters. Those scenarios represent different real-time conditions where obstacles differ from one scenario to another. For example, the line of sight scenario assumes there a clear line of sight between transmitter and receiver, Non line of sight assumes many obstacles between the transmitter and receiver, i.e. walls, cabinets, etc. and Two Wave Diffuse Power assumes a metallic reflector surrounding the receiver. The experiment showed more accurate results when compared to the one dimensional measurement that has been done in the past where 
receiver is moving in one direction and also receiver being fixed where a constructive and destructive interference is not captured. The two dimensional measurement technique, i.e. capturing data while receiver moving in both $x$ and $y$ directions, provided essential information regarding the constructive and destructive interference patterns caused by the interaction between the receiver while moving and the obstacles surrounding the receiver.

\section{Keywords}

Small-Scale Fading, Gaussian, Rayleigh, Rician, TWDP, Maximum Likelihood

\section{Introduction}

Accurately characterizing the wireless small-scale fading channel has been a challenging task in the wireless communication era. However, as difficult as this task appears to be, it did not prevent developers to always find ways to improve the accuracy of characterizing the wireless channel. Unlike large-scale fading, a small-scale fading experiences rapid fluctuations of the transmitted signal due to the propagation environment, i.e. different types of unpredictable obstructions between transmitter and receiver. Measurements of the small-scale channel have been done in the past as reported in [1]-[3], however there are major discrepancies between the propagation modeling of the channel and the actual measurements. The major discrepancies will be explained in detail in the next paragraphs.

Current small-scale fading measurement technologies are only able to offer a limited number of concrete measurements, which often is not enough data to assess complex small scale fading phenomenon. One-dimensional measurements have been studied in the past are reported in [1]. However, these studies only conducted measurements along one dimension and did not take under consideration that the signal would be traveling in a two-dimensional (2D) manner. By only conducting one-dimensional measurements essential components of the received signal strength variation, i.e. angle and distance from transmitter to receiver, are not part of the measurements. As a result, the measured data when compared with the model distributions did not seem to be adequate in describing small-scale fading for indoor propagation environments. Furthermore, due to the one dimensional measurement, there was break down for realistic situations where there are not enough data.

This task is principally illuminating a non-standard fading behavior in a close-in environments. This is very important for next-generation wireless systems, which are densified (increased cell density) and emerging Internet-of-things applications, where machines and objects will be connected wirelessly in the different types of environments. Some interesting two wave diffused power (TWDP) behavior, which has been measured in the past only sporadically by others. The TWDP is a particularly nasty distribution because of Delta $>1$, the relative magnitudes of the TWDP distribution and high $\mathrm{K}$, where $\mathrm{K}$ is the ratio of deterministic signal power to the average power of the rest of the signal, which indicates deep fades for the desired links but also frequent consecutive peaks (for interferers).

Researchers have considered measurements, estimation as reported in [4] and modeling of the propagation effects in the indoor environments; however, they have only considered a specific environment explicitly, i.e. the receivers are mounted in different locations in an indoor environment, and characterized them empirically using different modelling schemes as reported in [5]. The work presented in [5] only considered a fixed wireless devices environment and thus the empirical results and modeling cannot represent a mobile device environment, where the wireless communication takes place while the mobile device is moving. Furthermore, when the receiver is being mounted or fixed in a location, the measured data will not show much of the constructive and destructive interference which is caused by the obstacles within the room in relation to the movement of the wireless device i.e. receiver.

The work presented herein an alternative technique for measuring the small-scale fading that allows for the measurement of 2D field; wherein the measurements are taken at regular intervals as the wireless device is moving down the track in both $x$-axis and $y$-axis direction. In this paper, we investigate both constructive and destructive interference which is caused by the obstacles within the room in relation to the movement of the wireless device. Techniques offered in the paper will enable the receiver to capture more useful information, 
unlike work done in the past wherein the data captured is basically the variation in amplitude due to the distance variation in one direction only. Techniques in this paper will capture data due to angle variation, since the receiver is moving in two directions, constructive and destructive interferences caused by the obstacles within the room in relation to the movement of the receiver. Furthermore, different modeling schemes were investigated in the 2D environment as well as the movement of the wireless device.

\section{Test Setup}

An experiment was carried out to obtain the received level characteristics in a laboratory room on the top floor of the Van Leer building at the Georgia Institute of Technology. The room is approximately 7 meters long and 4 meters wide as shown in Figure 1. The environment inside the lab includes many obstacles between the transmitter and receiver (metal shelves, tables, chairs, and testing equipments, located mostly against the walls) as shown in Figure 3. The receiver is mounted on a three-dimensional positioner machine. The receiver moves towards and away from the transmitter along the $x$-axis, and along the $y$-axis. The movement of the linear positioner is in a two-dimensional manner. The linear positioner moves $45.7 \mathrm{~cm}$ along the $x$-axis and $y$-axis respectively. The movement of the linear positioner was programed to move along both the axis in $2.5 \mathrm{~cm}$ step size.

A signal generator was used to transmit signal at power levels $-5 \mathrm{dBm}$, and $15 \mathrm{dBm}$ at a carrier frequency of $2.43 \mathrm{GHz}$. A spectrum analyzer was used to receive the signal and record its level. Omni-directional antennas were used to transmit and receive the signal. The separation distance between the transmitter and receiver was different under various test scenarios. Furthermore, a heavy metal cabinet was used in one experiment and aluminum foil on a frame in another to act as large signal reflectors to create two specular components. In this experiment, three general scenario types were constructed.

Figure 1 shows a transmitter antenna and a receiver antenna separated by a $3 \mathrm{~m}$ distance. The transmitter antenna is connected to a signal

For simplicity of understanding, three-dimensional linear motion along the $x$-axis and $y$-axis will be considered in this paper. The location of the transmitter is known and is taken to be fixed relative to the mobile receiver. The mobile receiver is installed on the extended arms of the three-dimensional linear positioner, which can move its three mutually perpendicular arms in three dimensions. The entire system with receiver, transmitter, and positioner is illustrated in Figure 2. Signal measured in both $x$ and $y$ directions are sensed at the mobile receiver, while the mobile receiver is moving in $x$ and $y$ direction. The received signal amplitude which will be used to compute the approximate position of the receiver in $x$ and $y$ direction relative to the transmitter.

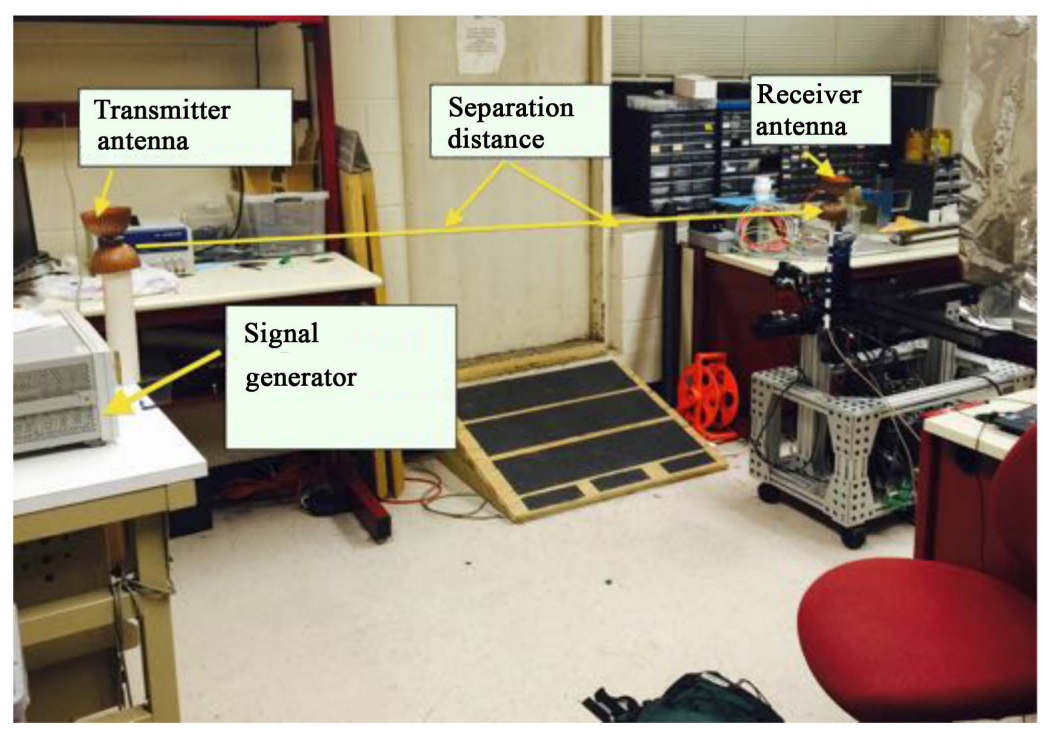

Figure 1. Test setup: Transmitter and receiver are separated by $3 \mathrm{~m}$. Transmitter antenna is connected to a signal generator, and receiver is mounted on an extended arm on a linear positioner, wherein the extended arm moves in both $x$ and $y$ directions. 


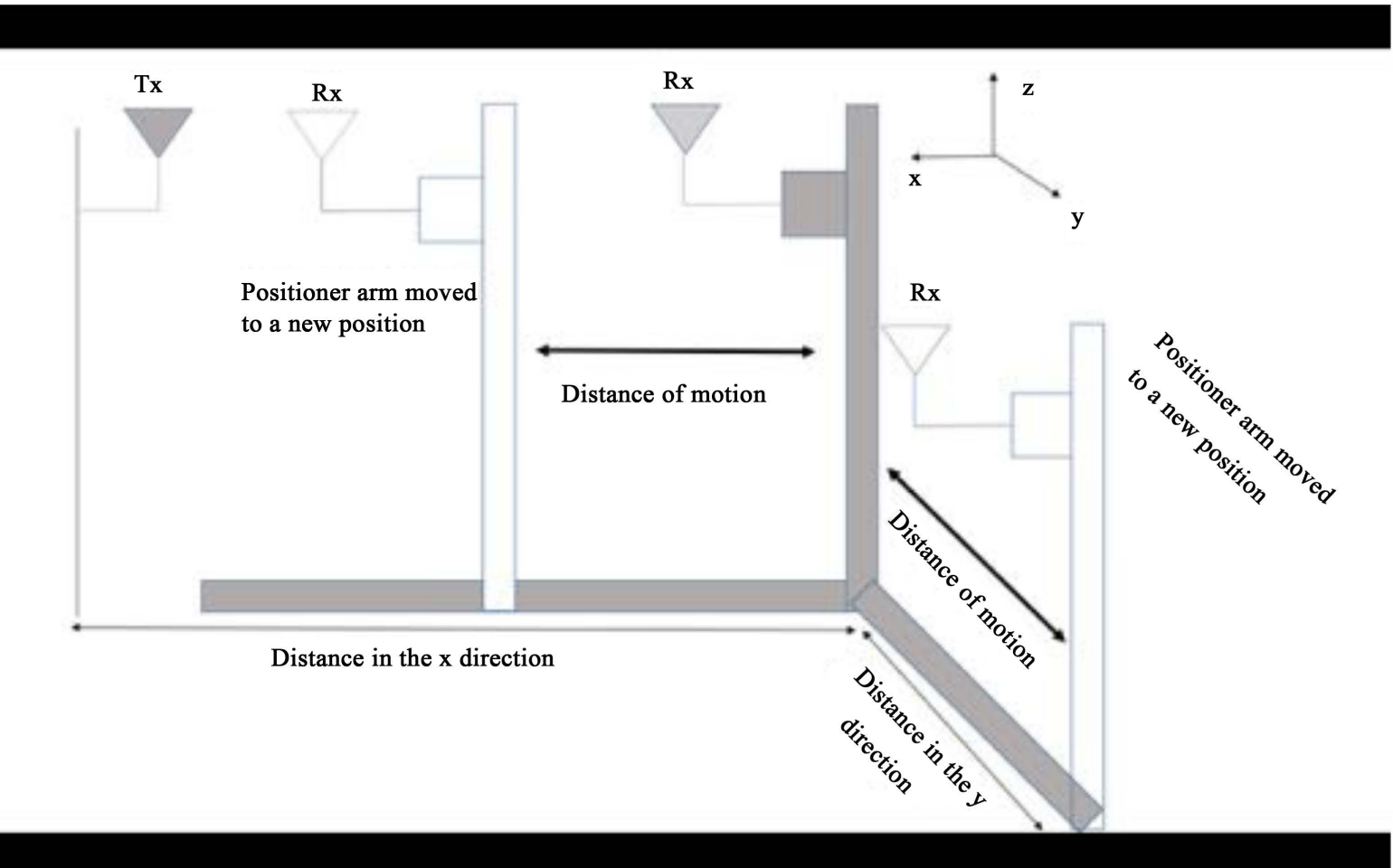

Figure 2. Three-dimensional positioner setup. The mobile receiver moves towards the transmitter along the $x$-axis and $y$-axis. The extended arm shows its positions after the positioner movement.

Figure 3 shows a receiver antenna, which receives the signal and is connected to a spectrum analyzer. The spectrum analyzer collects the raw data (at a given position in the $x$ and $y$ directions) and loads it into the computer. Also visible in the figure is a metal cabinet behind the linear positioner that is used as a reflector in one of the set-up.

\section{Test Scenarios}

Three scenarios were tested during the experiments as expressed below:

\subsection{Line-of-Sight (LOS)}

Some indoor environment scenarios generally assume the existence of line of sight link in addition to multipath, delayed, lower power reflections. Thus in this scenario, we investigate the possibility to establishing LOS links in an indoor environment area, i.e., where the line of sight is available. The first scenario was the line-of-sight (LOS), wherein a pre-determined separation distance, i.e. 3 meters, between the transmitter and receiver that is mounted on the linear positioner was selected. Multiple runs, i.e. ten to twenty runs, were conducted in order to have enough data to perform the analysis. In this scenario, there were no obstacles between the transmitter and receiver in order to receive a clear or a strong signal.

\subsection{Non-Line-of-Sight (nLOS)}

One main source of errors of multipath fading is non-line-of-sight (NLOS) propagation conditions. While the errors resulting from multipath channel fadings can be easily avoided using classical diversity schemes, the issue of NLOS propagation is not so straightforward. In indoor environments, due to large number of walls, obstacles and moving people, the first arrival path between a transmitter and a receiver is rarely LOS. The second scenario was the non-line-of-sight (nLOS), wherein the transmitter was put outside the room in order to create a pure multiple path environments. The path between the transmitter and receiver had obstacles, e.g. walls and metal cabinets. Due to the fact that the separation distance between the transmitter and receiver was further than the 


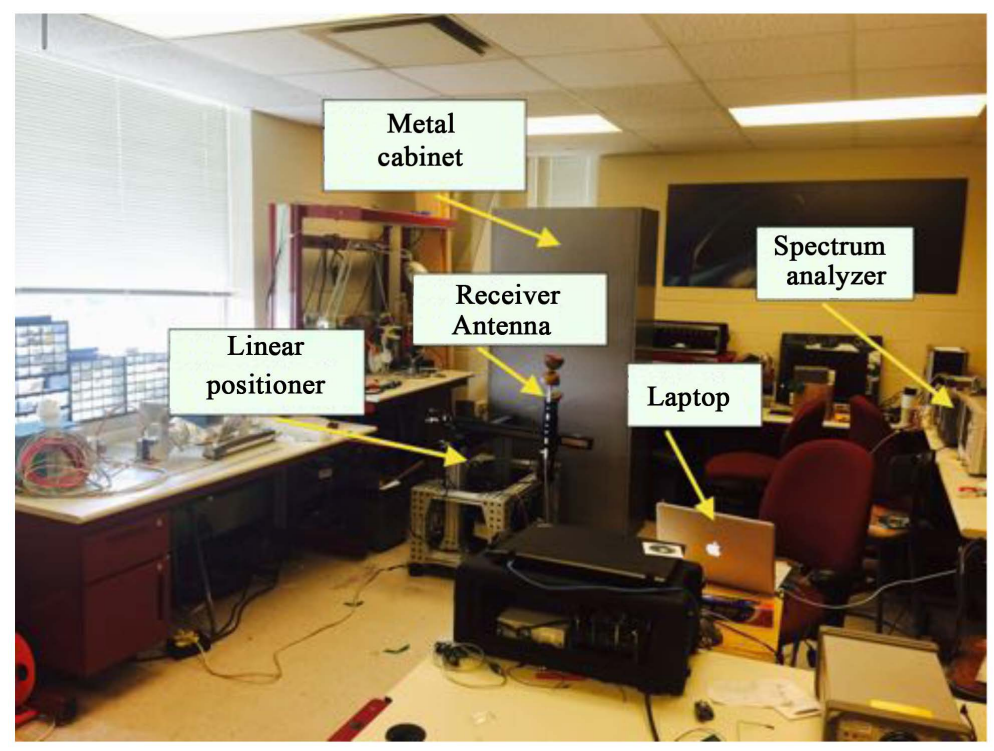

Figure 3. Test setup showing a metal cabinet used as reflector in one of the test scenarios and spectrum analyzer used to receive the transmitted signal. A receiver mounted on an extended arm attached to the linear positioner.

LOS scenario, different transmitted power levels were selected, i.e. between $-5 \mathrm{dBm}$ and $15 \mathrm{dBm}$, in order to compensate for the longer distance between the transmitter and receiver, i.e. longer than LOS scenario, as well as more and numerous types of obstacles.

\subsection{Two-Wave Diffuse Power}

A related distribution is the Two Wave with Diffuse Power (TWDP) fading model. This distribution can characterize a large range of fading behavior and has a geometric justification in terms of two dominant line of sight components in the presence of a diffuse component. This distribution consists of two LoS components and multiple diffuse NLoS components, wherein this scenario occurs when there are large metal reflector that reflects the transmitted signal in which as a result a two wave signal is created with two dominant line of sight components. The third scenario was Two-Wave Diffuse Power (TWDP). TWDP has a non-specular component with numerous individual waves, each carrying power that is negligible compared to the total average power of either of two specular components as reported in [1]. This scenario used the metal cabinet as a reflector as shown in Figure 3 for one data set. Another data set included an aluminum foil, which was used as a reflector, shown in Figure 4.

Furthermore, in Figure 5, where the linear positioner is shown, there is a big metallic block that carries the linear positioner, extended arms, and controller. When the linear positioner is at its origin i.e. $(0,0)$, the way the receiver is mounted is aligned with the front of the metallic block. The metallic block will have some effect on the results as will be explained later.

\section{Theory}

In this section we present three different distribution models, i.e. LOS, NLOS, and TWDP. These models are used later to compare the results of the measurements for each scenario. The result of the comparison between the models and the actual measurement data will indicate how accurate are the measurements in each scenario.

\subsection{Fading}

In wireless communication, fading is deviation of the attenuation of a signal over a propagation fading channel; wherein the fading channel is the communication channel that experiences fading. Furthermore, the fading is often modeled as a random process. Fading can be either due to multipath propagation as reported in [6] [7], which is referred as multipath fading. Also, shadowing from obstacles effecting the propagation signal is known as shadow fading. 


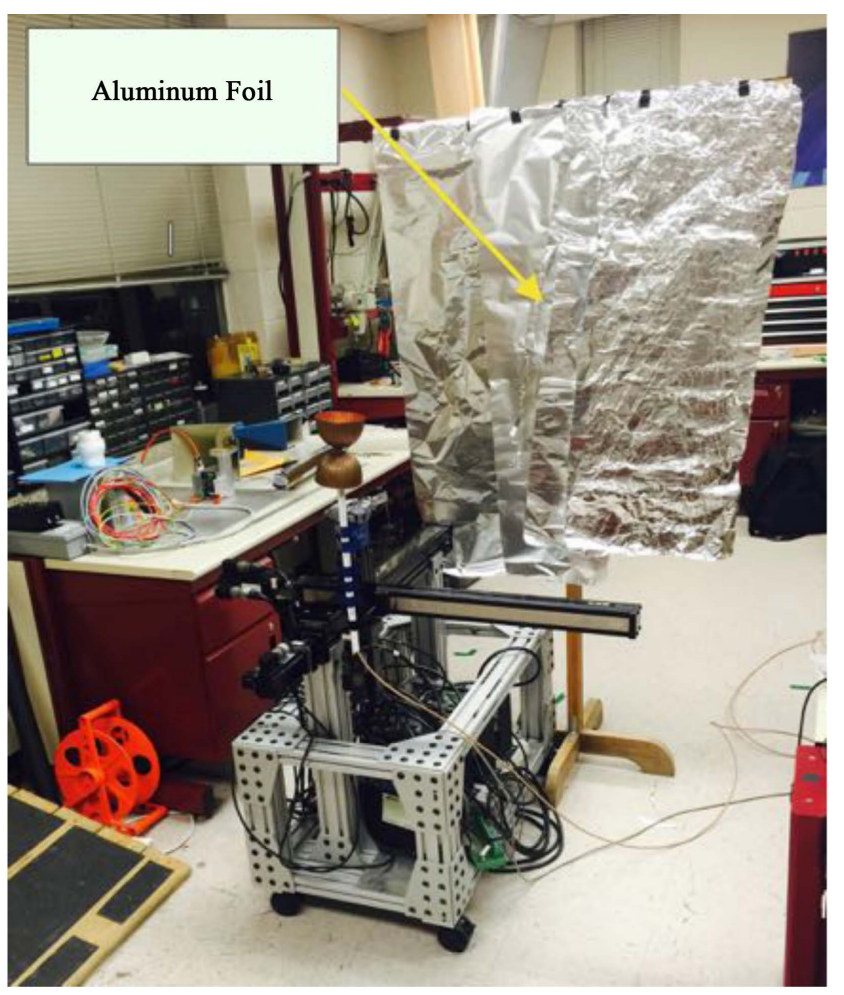

Figure 4. Test setup showing aluminium foil used as a reflector. Aluminum foil was investigated to test how strong is the reflectivity of the signal compared to the large metal cabinet.

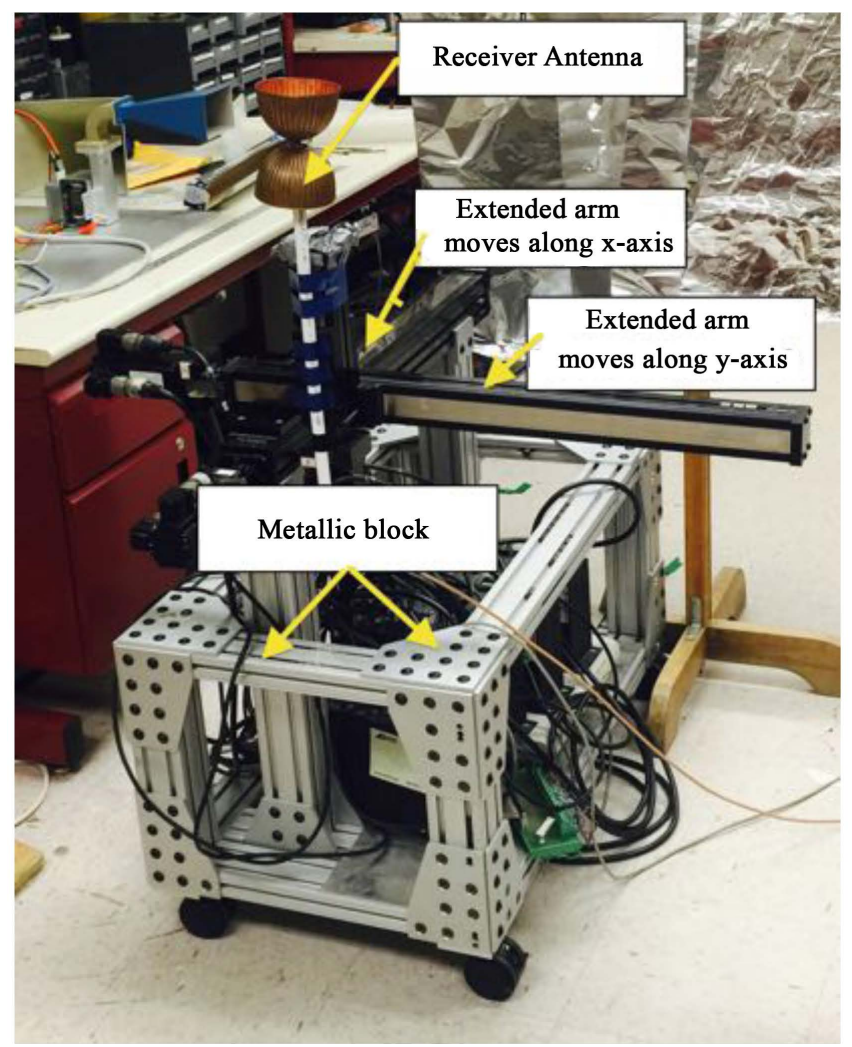

Figure 5. Metallic body of the positioner with an extended arm with the receiver antenna. 


\subsection{Interference}

There have been many investigations and experiments regarding indoor measurements in a systematic way at $2.4 \mathrm{GHz}$ as reported in [8]-[10]. The presence of reflectors in the environment surrounding a transmitter and receiver create multiple paths that a transmitted signal can traverse. As a result, the receiver sees multiple copies of the transmitted signal, each traversing a different path. Each signal copy will experience differences in attenuation, delay and phase shift while traveling from the source to the receiver. This can result in either constructive or destructive interference, amplifying or attenuating the signal power seen at the receiver.

\subsection{Background}

A given time variant signal $s$ is expressed in terms of real and imaginary parts given by:

$$
s=u+j v
$$

wherein $u$ and $v$ are independent random variables, and the magnitude $r$ and the phase $\phi$ of the time variant signal $s$ is given by:

$$
\begin{gathered}
r=|s|=\sqrt{u^{2}+v^{2}} \\
\phi=\arctan \frac{v}{u}
\end{gathered}
$$

The probability density function (pdf) for the Rayleigh Distribution random variable as reported in [1] is given by:

$$
f_{R}(r)=\frac{r}{\sigma^{2}} \exp \left(-\frac{r^{2}}{2 \sigma^{2}}\right)
$$

where $\sigma^{2}$ is the variance of the data.

\subsection{Rician Distribution}

A Rician distribution is given by:

$$
\sigma=\sqrt{u+v+A}
$$

where $A$ is a non-random component. Now in addition to the various non-LOS paths, there is a LOS path with peak amplitude $A$, where $K$ is the Rician factor is given by:

$$
K=\frac{\left|A^{2}\right|}{2 \sigma^{2}}
$$

where $K$ is the ratio of deterministic signal power to the average power of the rest of the signal. This is called K-factor or Rician Factor. When $K>1$, a deep fade is not expected. Rician pdf is given by [2]

$$
f_{R}(r)=\frac{r}{\sigma^{2}} \exp \left(-\frac{r^{2}+A^{2}}{2 \sigma^{2}}\right) I_{0}\left(\frac{A r}{\sigma^{2}}\right)
$$

where $I_{0}$ is the zeroth order modified Bessel function.

\subsection{Two Wave Diffused Power}

Recently there has been theoretical innovations in TWDP fading as reported in [11], where a convergent infinite series expressions for the TWDP fading pdf and cdf (cumulative distribution function) were derived. A diffuse component is a non-specular component with numerous individual waves, each carrying power that is negligible compared to the total average power of the diffuse component. The diffused component channel can be written in terms of specular and non-specular components as reported in [12] given by:

$$
\hat{V}=\sum_{i=1}^{N} V_{i} \mathrm{e}^{j \phi_{i}}+\sum_{i=1}^{M} V_{i} \mathrm{e}^{j \phi_{i}}
$$


where $N$ and $M$ are the number of the specular waves and the non-specular components respectively, $\hat{V}$ is estimated amplitude, $V$ is the amplitude and $\phi$ is the random phase. There is not exact TWDP pdf, however, the approximation TWDP pdf as reported in [12] is given by:

$$
f_{R}(r)=\frac{r}{\sigma^{2}} \mathrm{e}^{\left(-\frac{r^{2}}{2 \sigma^{2}}-K\right)} \sum_{i=1}^{N} \alpha_{i} D\left(\frac{r}{\sigma} ; K, \Delta \cos \frac{\pi(i-1)}{2 M-1}\right)
$$

where $\alpha$ are the coefficients while here the $K$ factor is, different than the $K$ factor for the Rician factor, given by:

$$
K=\frac{V_{1}^{2}+V_{2}^{2}}{2 \sigma^{2}}
$$

where $V_{1}$ and $V_{2}$ are the voltage magnitude of two specular components, and

$$
\begin{gathered}
\Delta=\frac{2 V_{1} V_{2}}{V_{1}^{2}+V_{2}^{2}} \\
D(x ; K, \alpha)=\frac{1}{2} \exp (\alpha K) I_{0}(x \sqrt{2 K(1-\alpha)})+\frac{1}{2} \exp (-\alpha K) I_{0}(x \sqrt{2 K(1+\alpha)})
\end{gathered}
$$

\subsection{Maximum Likelihood (Rician and TWDP)}

Both $A$ and $\sigma$ are correlated thus it is desired to simultaneously estimate both the quantities by maximizing the likelihood function as reported in [13], given by:

$$
\{\hat{A}, \hat{\sigma}\}=\arg \left\{\max _{A, \sigma}(\log (L))\right\}
$$

where $\hat{A}$, and $\hat{\sigma}$ are the estimated amplitude and variance. The maximum likelihood $L$ is given by:

$$
L(\theta ; r)=\prod_{i=1}^{N} f_{i}\left(r_{i}, \theta\right)
$$

where $f_{i}\left(r_{i}, \theta\right)$ is the pdf of underlying distribution and $\theta$ is the parameter to be estimated. However, it is easier to calculate the log likelihood function as it reduces the above equation to series of additions instead of multiplication of several of terms as reported in [13] shown below:

$$
\begin{gathered}
\ln L(\theta ; r)=\sum \ln \left(f_{i}\left(r_{i}, \theta\right)\right) \\
L=\prod_{i=1}^{N} \frac{M_{i}}{\sigma^{2}} \exp \left(-\frac{M_{i}^{2}+A^{2}}{2 \sigma^{2}}\right) I_{0} \frac{A M_{i}}{\sigma^{2}} \\
\log (L)=\sum_{i=1}^{N} \log \left(\frac{M_{i}}{\sigma^{2}}\right) I_{0} \frac{A M_{i}}{\sigma^{2}}-\left(\frac{N A^{2}}{\sigma^{2}}\right)-\sum_{i=1}^{N}\left(\frac{M_{i}}{2 \sigma^{2}}\right)
\end{gathered}
$$

\section{Results}

In this section, we will discuss the results for the three scenarios, Line-of-sight, Non-line-of-sight, and Two Wave Diffused Power. The analysis was performed using Matlab software. Furthermore, the result for each scenario consist of two figures. The first figure shows the distribution of the measured data versus the theoretical models. The second figure the two dimensional data, where the colors of the two dimensional graph show the constructive and destructive interference pattern, i.e. deep fading. The intensity of the received power is relatively high denoted by the red color. However, when the extended arm, i.e. receiver, moves away along the $\mathrm{x}$-axis and $\mathrm{y}$-axis. i.e. at an angular direction, the received power starts deteriorating, denoted by various intensity of yellow and blue colors.

\subsection{Line-of-Sight (LOS)}

The first part of the analysis is analyzing the measured data by comparing the measured data with the models. 
Figure 6 shows a histogram from a few representative data runs, with various probability density functions overlaid. Using the Maximum Likelihood estimator as reported in [13] to estimate the Rician distribution $K$ value which found to be $1 \mathrm{~dB}$. As shown in Figure 6, the histogram is approaching a Rician distribution with more than one specular component. Furthermore, the peaks are not weak to be diffused but are weak enough to show overlaid peaks on a Rician distribution. As expected, the histogram in Figure 6 almost fits the Rician distribution. However, the histogram shows a complex multi-peak structure, likely due to a combination of changing angle and amplitude with respect to both the transmitter and various reflectors in the room, e.g. metal cabinets. There were a few strong reflectors in the room, and conceivably one or more of them could have caused constructive and destructive interference. The second part of the analysis is to analyze the constructive and destructive interference patterns by analyzing the the two dimensional graph. Figure 7 represents a two dimensional data; however, it does not show clear evidence of a regular interference pattern, however, if this is what is occurring, different constructive interference peaks are likely due to different reflectors. It is noticed that at position $(0 \mathrm{~cm}, 40 \mathrm{~cm}$ to $45 \mathrm{~cm})$, where the extended arm of the linear positioner with the receiver mounted on it is at the closest position to the transmitter and aligned with the transmitter, the intensity of the received power is relatively high denoted by the red color. However, when the extended arm, i.e. receiver, moves away along the $x$-axis and $y$-axis. i.e. at an angular direction, the received power starts deteriorating, denoted by various intensity of yellow and blue colors. This phenomenon is due to the constructive and destructive interference. Furthermore, the area where $x$-axis is between about $0 \mathrm{~cm}$ and $26 \mathrm{~cm}$ and $y$-axis is between about $20.32 \mathrm{~cm}$ and $45.72 \mathrm{~cm}$ has high peaks because the receiver is closest to the transmitter and also the receiver is aligned with transmitter, as a result, clear line of sight is created. Fading in various areas is due to the chaotic effect due the surrounding environment as well as the angular movement of the receiver.

If circles to be drawn around the high peaks, denoted by red colors, starting from left top corner, it is noticed that the power starts fading out gradually as the receiver moves away from the transmitter both in the $y$-axis and $x$-axis direction, i.e. at an angular direction. Furthermore, there is a void in the $x$-axis around $10 \mathrm{~cm}$ to $30 \mathrm{~cm}$ and in the $y$-axis around $5 \mathrm{~cm}$ to $18 \mathrm{~cm}$, denoted by yellow and blue colors, and that is due to a chaotic effect. If the test was conducted in a larger area, a gradual fallout would be observed which is what the Rician distribution is, but this test was restricted to a smaller area. A gradual fall-out is realized at $x$-axis around $7 \mathrm{~cm}$ and $y$-axis around $45 \mathrm{~cm}$ and diagonally down to the right.

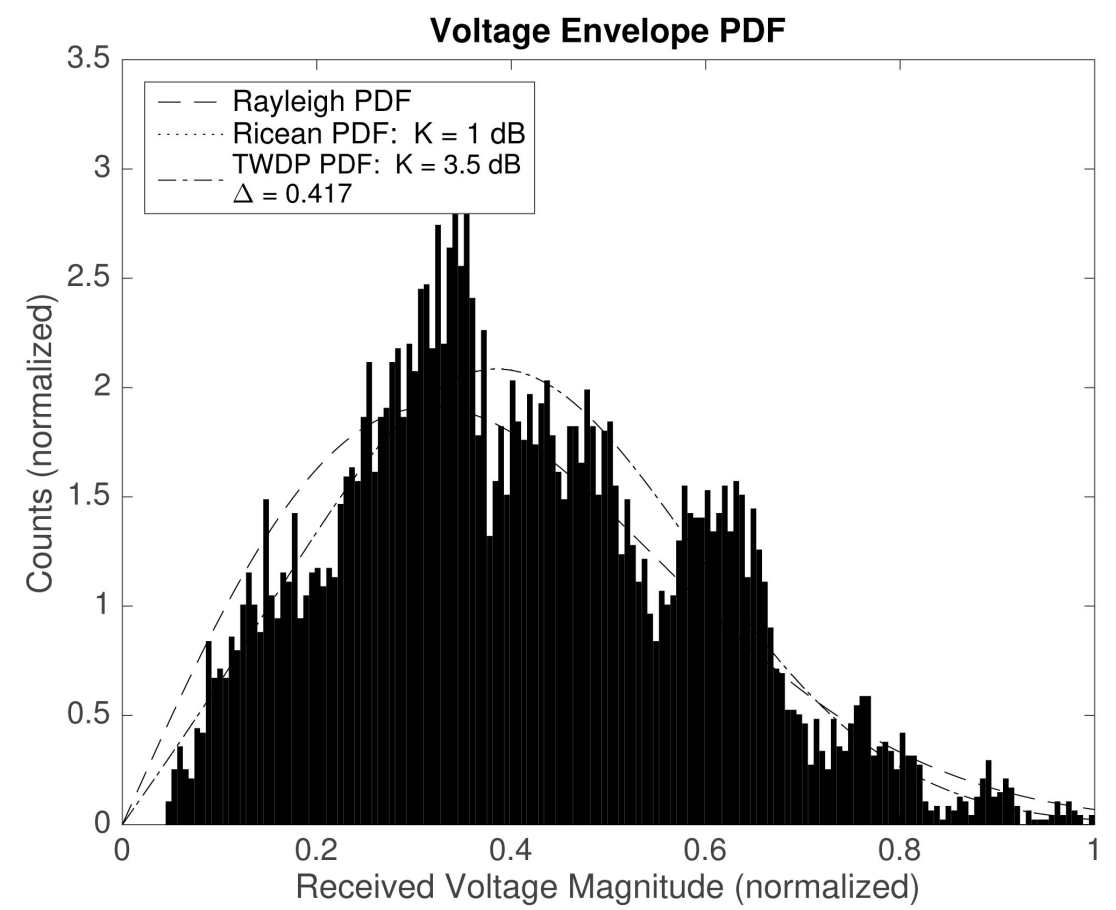

Figure 6. Histogram of measured voltage magnitude at the receiver with overlaid pdf models. Note that the Rician pdf follows the histogram. 


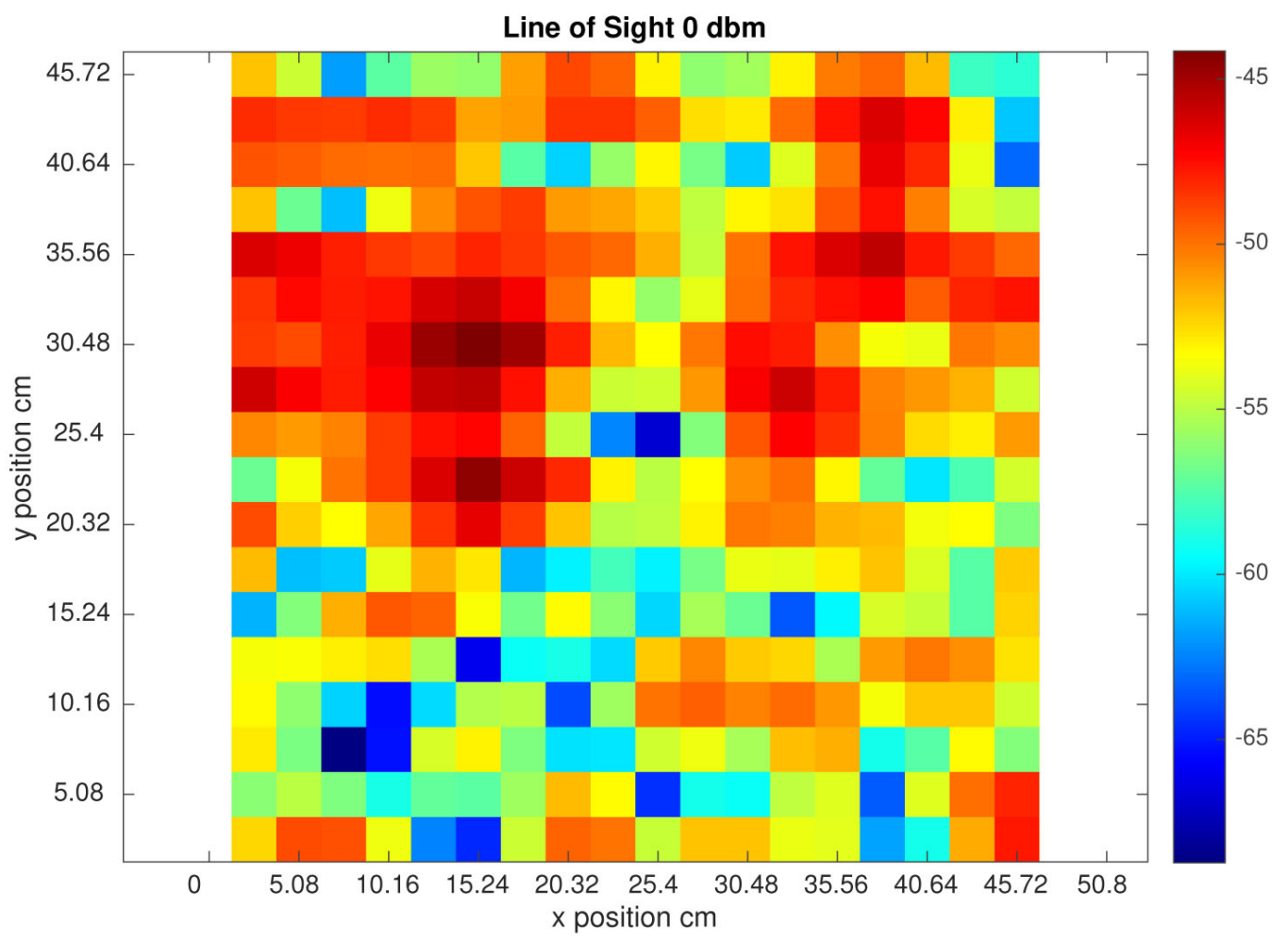

Figure 7. A two-dimensional representation of the received voltage with respect to a position along the $x$-axis and $y$-axis. Wherein colors shown in the graph represent highest to lowest power level in the following order: Red, Yellow, Light Blue, and Dark Blue.

In one-dimensional measurements, these multiple peaks would likely be averaged out into one or two peaks previous work as reported in reference [1] supports this, as one dimensional measurements by those groups did not see a set of peaks like this. Thus, this is likely to be an effect that only shows up in higher dimensions, i.e. two-dimensional.

\subsection{Non-Line-of-Sight (nLOS)}

Figure 8 shows an excellent fit for the Rayleigh distribution in which there was no clear line of sight signal between the transmitter and receiver, i.e. many obstructions were blocking the signal between the transmitter and receiver, e.g. a door, metal cabinets, and walls.

Figure 9, a two dimensional graph, shows a chaotic distribution, i.e. a random behavior of received signal strength, as expected. Regarding non-LOS scenario, it is noticed that there was no clear evidence of a constructive and destructive interference pattern. This phenomenon is expected because of the various types of obstacles between the transmitter and receiver. In the non-LOS scenario, the obstacles between the transmitter and receiver are walls, metal cabinets, and other equipments inside the room, wherein the transmitter was placed outside the room in the hallway. In a non-LOS phenomenon, both a constructive and destructive interference occur; however based on Figure 9, we notice more destructive interference, which is justified by the obstacles that were present between the receiver and transmitter. Furthermore, we notice that there are many blue and yellow zones, i.e. low peaks, which is due to the far distance between the receiver and transmitter and also the number of obstacles between the transmitter and receiver in which the transmitted power weakens.

\subsection{Two-Wave Diffuse Power (TWDP)}

Figure 10 shows the data when an aluminum foil reflecting sheet was set up behind the receiver track. As shown in the graph, the histogram almost approaches a TWDP distribution except the TWDP distribution does not show the two peaks. We suspect that the reason the two peaks are not shown in this distribution is because 


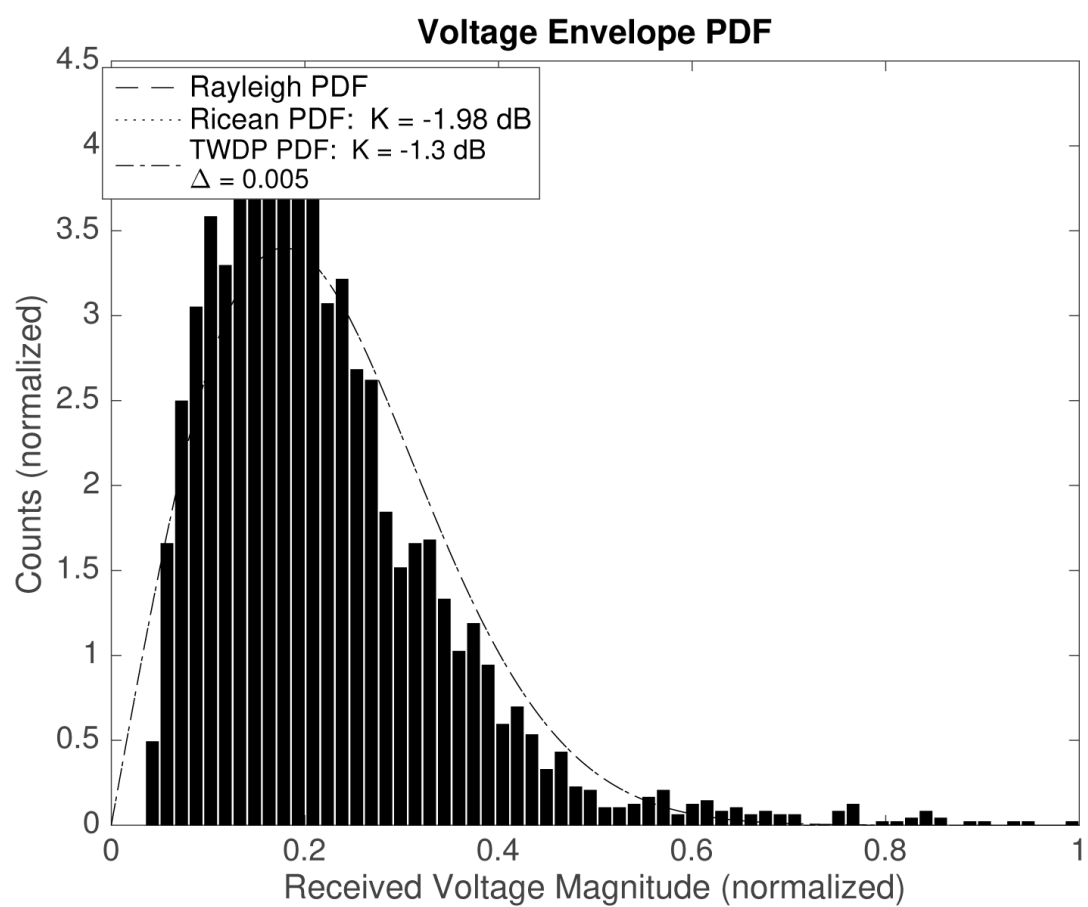

Figure 8. Histogram of measured voltage magnitude at the receiver. Note that the Rayleigh pdf follows the histogram.

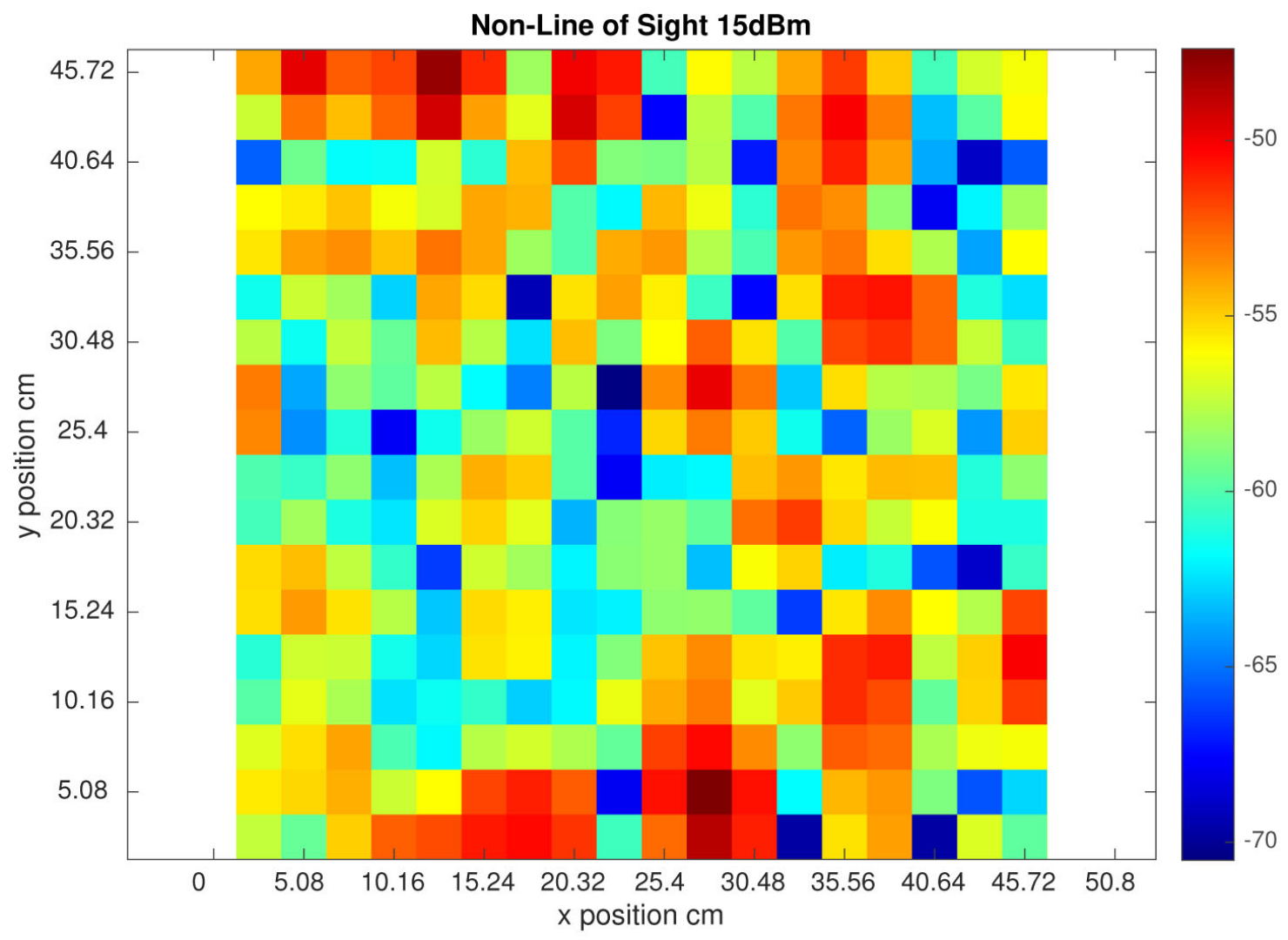

Figure 9. A two-dimensional representation of the received voltage with respect to a position along the $x$-axis and $y$-axis. Wherein colors shown in the graph represent highest to lowest power level in the following order: Red, Yellow, Light Blue, and Dark Blue. 


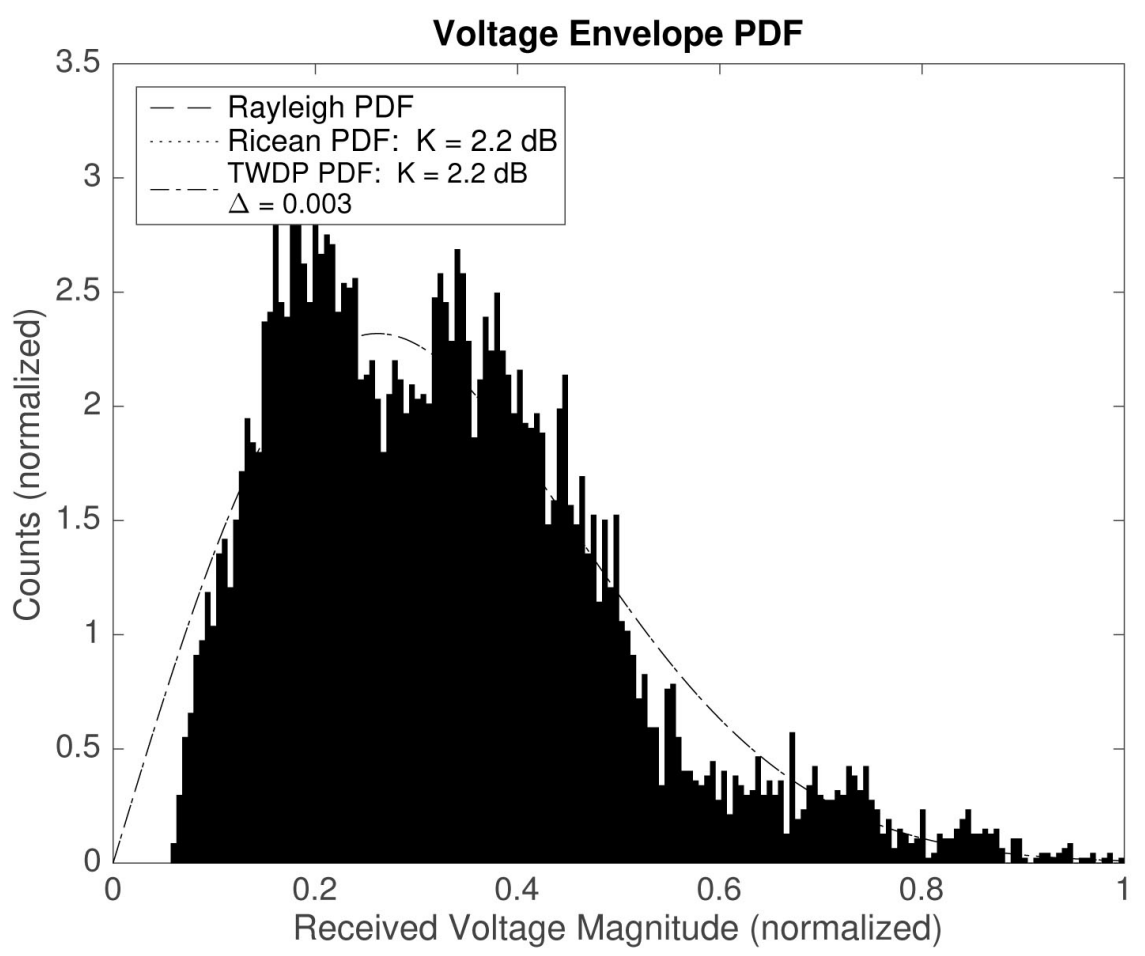

Figure 10. Histogram of measured voltage magnitude at the receiver. Note that the TWDP pdf follows the histogram as expected.

we did not account for the fact that the interference may follow a strict pattern based on the difference in path length. If true, the phases of the two specular components are not random with respect to each other, as assumed by [3].

Supporting this hypothesis, in Figure 11, there is a constructive interference pattern and there is a deconstructive interference pattern, i.e. a deep fade. Now the angular movement of the receiver is another factor that is causing an interference. It is noticed that the high peaks at the $x$-axis from $0 \mathrm{~cm}$ to $15 \mathrm{~cm}$ and along the $y$-axis, wherein the deep fade is within the high peaks. Furthermore, the high peaks are fairly uniformly spaced interference pattern. When the $x$-axis is from $15.24 \mathrm{~cm}$ to $45.72 \mathrm{~cm}$ along the $y$-axis in which the receiver is considerably too far from the transmitter, a chaotic effect occurs, and thus a specular component is weaker and chaotic effect is more significant. There is similar pattern high peak at the right side but weaker than the right side due to the fact that the right side is further from the transmitter than the left side. Thus, If the right side were to be normalized, a similar pattern as the left side will be realized, i.e. uniformly high peaks denoted by red color.

Closer to the reflector, the spot size diminishes, as the receiver detects less of the diffusion of the reflected wave. As the receiver moves along the $y$-axis, constructive and destructive interference still seems to appear, only as diffused streaks. The transmitter, receiver, and reflector now do not lie along a line, so the angle of reflection has an effect, changing the path length difference as well as introducing a non-180 degree phase change as reported in [14]. Thus, if you are at the right distance from a reflector, the statistical model for measuring the signal breaks down because the primary and reflected specular components have predictable phase with respect to one another and not random phase as assumed in the TWDP model.

Figure 12 shows a Two Wave Diffuse Power, which is similar to Figure 11, however, this time a metallic cabinet was used as a reflector instead. We notice that the constructive and destructive pattern is not as strong as the one with the aluminum foil. This is due to the metal cabinet not being as strong reflector as the aluminum foil. In other words, there is a loss of signal most likely through absorption. We notice as the receiver moves away from the metal block, a pattern of constructive and destructive interference occurs, i.e. red zones and blue zones. However, there are not many red zones, i.e. high peaks, which is due to the fact that the metal cabinet not being a strong reflector, i.e. there is loss in signal most likely through absorption. 


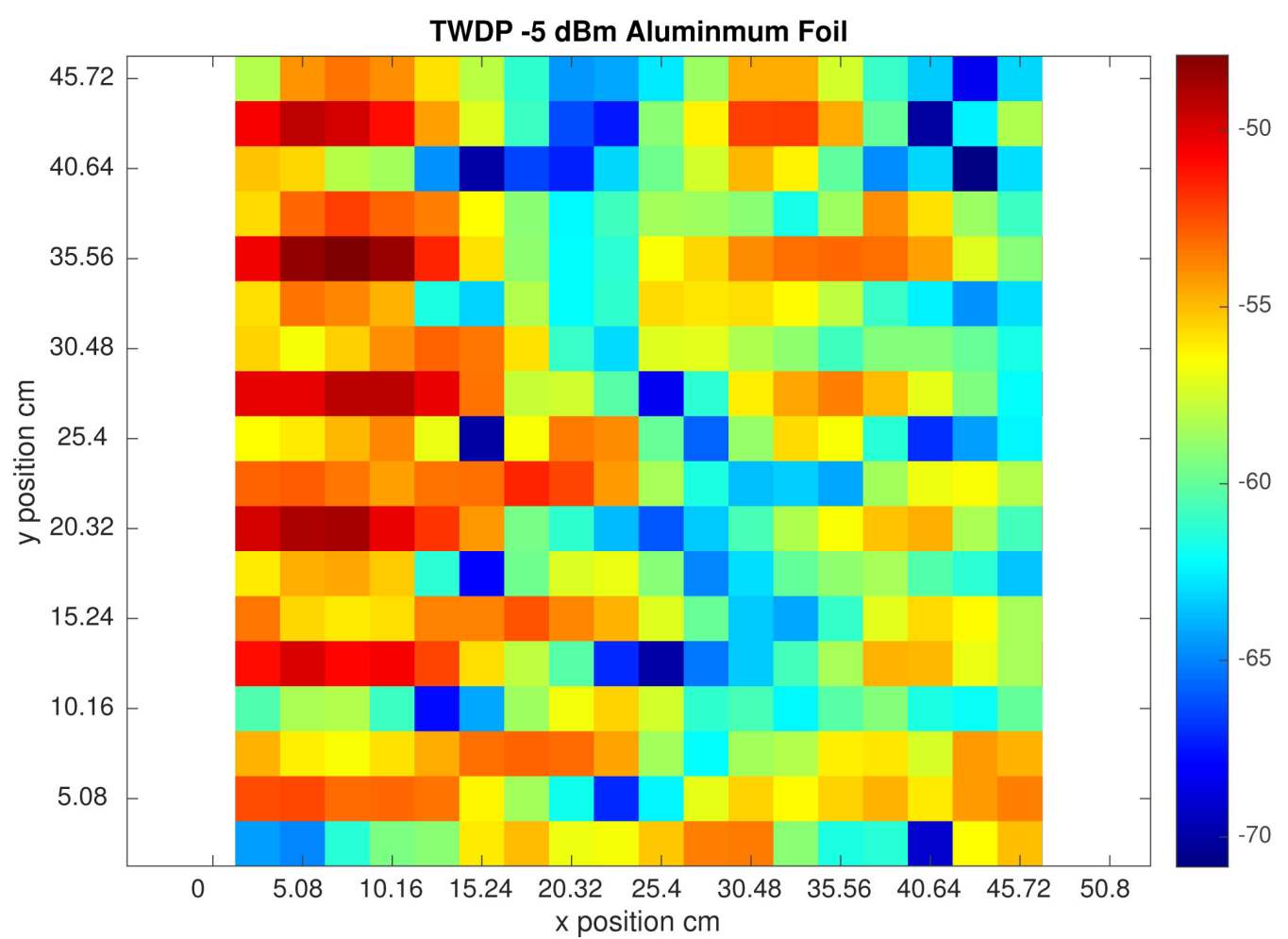

Figure 11. Test setup: Transmitter and receiver are separated by $3 \mathrm{~m}$. Wherein colors shown in the graph represent highest to lowest power level in the following order: Red, Yellow, Light Blue, and Dark Blue.

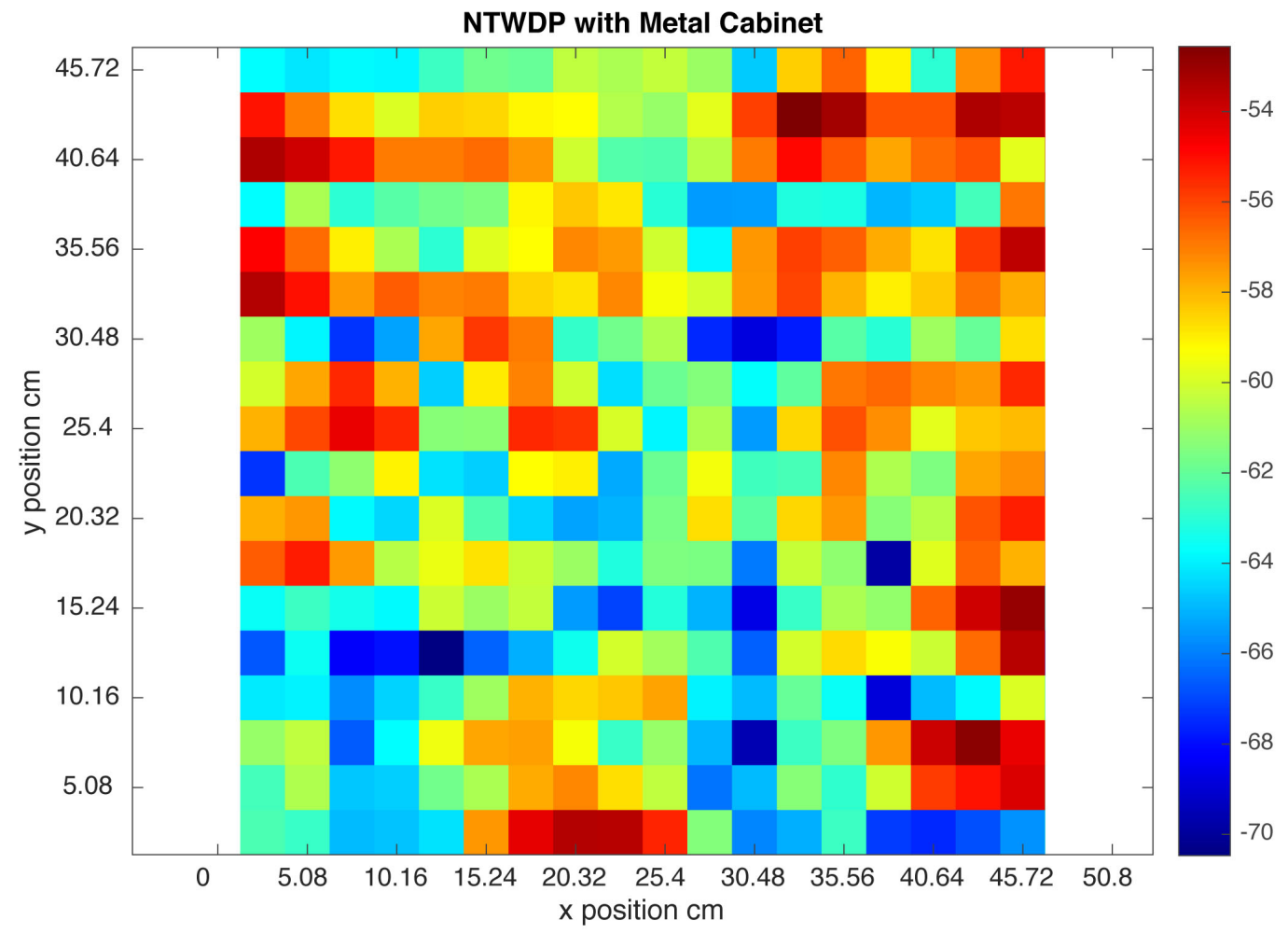

Figure 12. Test setup: Transmitter and receiver are separated by $3 \mathrm{~m}$. Wherein colors shown in the graph represent highest to lowest power level in the following order: Red, Yellow, Light Blue, and Dark Blue. 


\section{Conclusion}

This paper has presented a new measurement technique, 2D measurements while the receiver is in motion in both $x$ and $y$ directions, in which an improved characterization of the small-scale fading 2D was achieved. A detailed two dimensional measurement campaign is conducted for capturing the impact of the variations in the received signal envelope over an indoor wireless channel. We conducted measurements based on three scenarios, LOS, NLOS, and TWPD. From the measured data, we extracted individual distributions of small scale fading, LOS, NLOS, TWDP. Next we used a Rician distribution, i.e. LOS, NLOS, and TWDP to approximate the small scale fading distribution. Then we compared said distributions to their theoretical models. We also analyzed the constructive, destructive interference patterns utilizing the two dimensional data captured from the received signal. The 2D measurements showed data for Rayleigh and Rician scattering that better match theoretical models than previous 1D measurements. In particular, the non-line-of-sight data was very close to the theoretical Rayleigh distribution, while previous 1D measurements had a greater root-mean-squared error between model and data. In addition, an attempt to construct a Two Wave Diffuse Power distribution, which showed regularly, spaced peaks in certain regions of the measurement plane, consistent with non-random interference of the primary and reflected specular components. Based on these $2 \mathrm{D}$ measurements, we can conclude that the Rayleigh model is likely to be the best when there is no line of sight between transmitter and receiver. However, if there is such a line of sight, a Rician distribution may not adequately represent reality due to metal objects, which reflect radio waves being common in most workplaces. Receivers close to such objects, for instance a laptop near a metal cabinet, may even experience interference of components with non-random phase, at which point the traditional statistical models break down.

\section{References}

[1] Henderson, A., Durkin, C. and Durgin, G. Measurement of Small-Scale Fading Distributions in a Realistic 2.4 ghz Channel. https://smartech.gatech.edu/bitstream/handle/1853/36951/PGTR070815AHH.pdf

[2] Hashemi, H. (1993) The Indoor Radio Propagation Channel. Proceedings of the IEEE, 81, 943-968. http://dx.doi.org/10.1109/5.231342

[3] Hashemi, H., McGuire, M., Vlasschaert, T. and Tholl, D. (1994) Measurements and Modeling of Temporal Variations of the Indoor Radio Propagation Channel. IEEE Transactions on Vehicular Technology, 43, 733-737. http://dx.doi.org/10.1109/25.312774

[4] Kapetanovic, A., Mawari, R. and Zohdy, M.A. (2016) Second-Order Kalman Filtering Application to Fading Channels Supported by Real Data. Journal of Signal and Information Processing, 7, 61. http://dx.doi.org/10.4236/jsip.2016.72008

[5] Frolik, J. (2007) A Case for Considering Hyper-Rayleigh Fading Channels. IEEE Transactions on Wireless Communications, 6, 1235-1239. http://dx.doi.org/10.1109/TWC.2007.348319

[6] Hasimbegovic, E. and Kocan, E. (2014) Experimental Results for Statistical Properties of Signal Envelope in Indoor Fading Channel at 2.4 ghz. Telecommunications Forum Telfor (TELFOR), 230-233.

[7] Vinogradov, E., Joseph, W. and Oestges, C. (2014) Modeling and Simulation of Fast Fading Channels in Indoor Peer-to-Peer Scenarios. 8th European Conference on Antennas and Propagation (EuCAP), 433-434. http://dx.doi.org/10.1109/eucap.2014.6901784

[8] De Coster, I., Van Lil, E., Van de Capelle, A. and Perez-Fontan, F. (1999) Indoor Penetration and Interference Studies. IEEE VTS 50th Vehicular Technology Conference, 4, 2303-2307. http://dx.doi.org/10.1109/vetecf.1999.797349

[9] Mao, Y., Zhao, Z. and Jia, X. (2011) Understanding the Indoor Interference between IEEE 802.15. 4 and IEEE 802.11 b/g via Measurements. IEEE International Conference on Wireless Communications and Signal Processing (WCSP), $1-5$.

[10] Wong, A.H., Neve, M.J. and Sowerby, K.W. (2005) Performance Analysis for Indoor Wireless Systems Employing Directional Antennas in the Presence of External Interference. IEEE Antennas and Propagation Society International Symposium, 1, 799-802. http://dx.doi.org/10.1109/aps.2005.1551444

[11] Saberali, S. and Beaulieu, N. (2013) New Expressions for TWDP Fading Statistics. IEEE Wireless Communications Letters, 2, 643-646. http://dx.doi.org/10.1109/WCL.2013.090313.130541

[12] Durgin, G., Rappaport, T. and de Wolf, D.A. (2002) New Analytical Models and Probability Density Functions for Fading in Wireless Communications. IEEE Transactions on Communications, 50, 1005-1015. http://dx.doi.org/10.1109/TCOMM.2002.1010620 
[13] Sijbers, J., Dekker, A., Dyck, D. and Raman, E. (1998) Estimation of Signal and Noise from Rician Distributed Data. IEEE Antennas and Propagation Society International Symposium, 140-142.

[14] Dubois, A. (2004) Effects of Phase Change on Reflection in Phase-Measuring Interference Microscopy. Applied Optics, 43, 1503-1507. http://dx.doi.org/10.1364/AO.43.001503

Submit or recommend next manuscript to SCIRP and we will provide best service for you:

Accepting pre-submission inquiries through Email, Facebook, LinkedIn, Twitter, etc.

A wide selection of journals (inclusive of 9 subjects, more than 200 journals)

Providing 24-hour high-quality service

User-friendly online submission system

Fair and swift peer-review system

Efficient typesetting and proofreading procedure

Display of the result of downloads and visits, as well as the number of cited articles

Maximum dissemination of your research work

Submit your manuscript at: http://papersubmission.scirp.org/ 